\title{
Unravelling the contribution of complex trauma to psychopathology and cognitive deficits: a cohort study
}

Stephanie J. Lewis, Karestan C. Koenen, Antony Ambler, Louise Arseneault, Avshalom Caspi, Helen L. Fisher, Terrie E. Moffitt and Andrea Danese

\section{Background}

Complex traumas are traumatic experiences that involve multiple interpersonal threats during childhood or adolescence, such as repeated abuse. These traumas are hypothesised to cause more severe psychopathology and poorer cognitive function than other non-complex traumas. However, empirical testing has been limited to clinical/convenience samples and cross-sectional designs.

\section{Aims}

To investigate psychopathology and cognitive function in young people exposed to complex, non-complex or no trauma, from a population-representative longitudinal cohort, and to consider the role of pre-existing vulnerabilities.

\section{Method}

Participants were from the Environmental Risk Longitudinal Twin Study, a population-representative birth cohort of 2232 British children. At age 18 years (93\% participation), we assessed lifetime exposure to complex and non-complex trauma, past-year psychopathology and current cognitive function. We also prospectively assessed early childhood vulnerabilities: internalising and externalising symptoms at 5 years of age, IQ at 5 years of age, family history of mental illness, family socioeconomic status and sex.

\section{Results}

Participants exposed to complex trauma had more severe psychopathology and poorer cognitive function at 18 years of age, compared with both trauma-unexposed participants and those exposed to non-complex trauma. Early childhood vulnerabilities predicted risk of later complex trauma exposure, and largely explained associations of complex trauma with cognitive deficits, but not with psychopathology.

\section{Conclusions}

By conflating complex and non-complex traumas, current research and clinical practice underestimate the severity of psychopathology, cognitive deficits and pre-existing vulnerabilities linked with complex trauma. A better understanding of the mental health needs of people exposed to complex trauma could inform the development of new, more effective interventions.

\section{Keywords}

Childhood experience; comorbidity; epidemiology; trauma; aetiology.

\section{Copyright and usage}

(C) The Author(s), 2021. Published by Cambridge University Press on behalf of the Royal College of Psychiatrists. This is an Open Access article, distributed under the terms of the creative Commons Attribution licence (http://creativecommons.org/ licenses/by/4.0/), which permits unrestricted re-use, distribution, and reproduction in any medium, provided the original work is properly cited.
Exposure to trauma - namely, an event that involves danger of death, serious injury or sexual violation ${ }^{1}-$ is an important risk factor for mental illness. Descriptions of trauma-related mental illness largely originated from investigations of people exposed to traumas that occurred in adulthood (e.g. military combat) and/or in single instances (e.g. disasters). These descriptions led to the definition of post-traumatic stress disorder (PTSD), ${ }^{2}$ which informs assessment and treatment after all traumas. ${ }^{3}$ However, clinical observations have suggested that more 'complex' types of trauma - namely, a traumatic experience involving multiple events with interpersonal threats during childhood or adolescence (e.g. repeated child abuse) - might result in more severe outcomes than other 'non-complex' traumas. ${ }^{4-7}$ These clinical observations have become very influential in clinical practice; for instance, leading to proposed new diagnoses linked with complex trauma exposure, such as complex PTSD. ${ }^{5,8,9}$ Yet, they have not been comprehensively tested in population studies, leaving important knowledge gaps in the literature.

\section{Psychopathology and cognitive deficits}

It is unclear if people exposed to complex trauma have more severe psychopathology and cognitive deficits than those exposed to noncomplex trauma, or trauma-unexposed peers. Although a small number of studies have found that complex trauma is associated with broader psychopathology and poorer cognitive function than non-complex trauma, ${ }^{10-13}$ these studies focused on a restricted range of outcomes and used clinical/convenience samples. Other studies have investigated exposures that, in some cases, overlap with the construct of complex trauma (e.g. child victimisation, maltreatment or adverse childhood experiences), and have found that these exposures are also associated with psychopathology and cognitive deficits. ${ }^{14,15}$ However, because in other cases such exposures do not overlap with the construct of complex trauma (as shown in Supplementary Fig. 1 available at https://doi.org/10.1192/bjp.2021.57), the relevance of those findings to complex trauma is unclear. Furthermore, there has been no direct comparison of such exposures with other potentially traumatic events that would be classified as noncomplex traumas. Previous studies are therefore unlikely to have fully captured the psychopathology and cognitive deficits that are uniquely linked with complex trauma in the population.

\section{Possible underlying mechanisms}

It is also unclear why complex trauma exposure may be associated with more severe psychopathology and cognitive deficits. The dominant hypothesis is that complex trauma exerts more detrimental effects on the brain than non-complex trauma, because of repeated 
activation of the stress response during sensitive developmental periods, giving rise to more severe psychopathology and cognitive deficits. ${ }^{16}$ An alternative hypothesis is that individuals exposed to complex trauma have greater pre-existing vulnerability, which may confound the associations of complex trauma with psychopathology and cognitive deficits. ${ }^{15}$ Although experimental research using animal models has shown that early-life stress can have causal effects on later behaviours, most human research undertaken to test the translational validity of these findings has been cross-sectional, lacking pre-trauma assessments or genetically sensitive designs needed to investigate the role of pre-existing vulnerabilities. ${ }^{17}$ A small number of studies of victimisation have tested for confounding by pre-existing vulnerabilities, using longitudinal methods that accounted for pre-victimisation measures or twin designs that accounted for genetic and family-wide environmental factors, and have found mixed results. ${ }^{14,15,18,19}$ However, no studies of complex trauma have assessed the role of pre-existing vulnerabilities to date.

In this study, we aimed to address these knowledge gaps. To better understand psychopathology and cognitive deficits linked with complex trauma, we investigated differences across a broad range of measures of psychopathology and cognitive function in young people exposed to complex, non-complex and no trauma, in a large population-representative cohort of twins. To explore the origins of these presentations, we investigated the role of preexisting vulnerabilities measured prospectively in early childhood, and undertook twin-difference analyses.

\section{Method}

\section{Sample}

Participants were members of the Environmental Risk (E-Risk) Longitudinal Twin Study, which tracks the development of a birth cohort of 2232 British children. The sample was drawn from a larger birth register of twins born in England and Wales in 1994$1995 .^{20}$ Full details about the sample are reported elsewhere. ${ }^{21}$ Briefly, the E-Risk sample was constructed in 1999-2000, when 1116 families (93\% of those eligible) with same-sex 5-year-old twins participated in home visit assessments. This sample comprised 56\% monozygotic (MZ) and 44\% dizygotic (DZ) twin pairs; sex was evenly distributed within zygosity (49\% male). Families were recruited to represent the UK population of families with new-borns in the 1990s, on the basis of residential location throughout England and Wales and mother's age. Teenaged mothers with twins were overselected to replace high-risk families who were selectively lost to the register through non-response. Older mothers having twins via assisted reproduction were underselected to avoid an excess of well-educated older mothers. The study sample represents the full range of socioeconomic conditions in the UK, as reflected in the families' distribution on a neighbourhood-level socioeconomic index (called ACORN (A Classification of Residential Neighbourhoods), developed by CACI Inc. for commercial use in Great Britain): $:^{22} 25.6 \%$ of E-Risk families live in 'wealthy achiever' neighbourhoods compared with $25.3 \%$ nationwide; $5.3 \%$ live in 'urban prosperity' neighbourhoods compared with $11.6 \%$ nationwide; $29.6 \%$ live in 'comfortably off neighbourhoods compared with $26.9 \%$ nationwide; $13.4 \%$ live in 'moderate means' neighbourhoods compared with $13.9 \%$ nationwide; and $26.1 \%$ live in 'hard-pressed' neighbourhoods compared with $20.7 \%$ nationwide. E-Risk underrepresents 'urban prosperity' neighbourhoods because such households are likely to be childless.

Follow-up home visits were conducted when the children were aged 7 (98\% participation), 10 (96\% participation), 12 (96\% participation) and 18 (93\% participation) years. Home visits at ages 5, 7, 10 and 12 years included assessments with participants and their mother (or primary care-taker); the home visit at age 18 years included interviews only with participants. Each twin participant was assessed by a different interviewer. There were 2066 children who participated in the E-Risk assessments at age 18 years, and the proportions of MZ (55\%) and male same-sex (47\%) twins were almost identical to those found in the original sample at age 5 years. The average age of the twins at the time of assessment was 18.4 years (s.d. 0.36); all interviews were conducted after their 18 th birthday. The study sample at age 18 years was equally distributed across all deciles of the Index of Multiple Deprivation 2015, which measures relative levels of deprivation in small areas in England (Supplementary Fig. 2). There were no differences between those who did and did not take part at age 18 years, in terms of socioeconomic status assessed when the cohort was initially defined $\left(\chi^{2}=0.86, P=0.65\right)$, internalising or externalising symptoms at age 5 years $(t=0.40, P=0.69$ and $t=0.41, P=0.68$, respectively) or IQ scores at age 5 years $(t=0.98, P=0.33)$.

We assert that all procedures contributing to this work comply with the ethical standards of the relevant national and institutional committees on human experimentation and with the Helsinki Declaration of 1975, as revised in 2008. All procedures were approved by the Joint South London and Maudsley and the Institute of Psychiatry Research Ethics Committee (reference 1997/122). Parents gave written informed consent, and twins gave assent between 5-12 years and then written informed consent at age 18 years.

\section{Trauma exposure}

Trauma exposure was assessed at age 18 years, during private interviews. Participants were asked whether they had been exposed to trauma during their lifetime, according to DSM-5 PTSD criterion A. ${ }^{1}$ Participants who reported trauma exposure were then asked to describe the traumas they had experienced. We reviewed these descriptions, alongside information gathered in the Juvenile Victimization Questionnaire, Second Revision, adapted as a clinical interview, ${ }^{23}$ to identify participants who had been exposed to complex trauma and non-complex trauma. We defined complex trauma exposure as (a) a traumatic experience that involved (b) multiple events that were (c) interpersonal assaults or threats and (d) occurred in childhood or adolescence. These criteria were selected because they have been consistently highlighted as key elements for the definition of complex trauma in previous literature, supported by evidence suggesting that each criterion considered alone is associated with psychopathology or cognitive deficits. ${ }^{4-7}$ Examples of complex traumas included repeated child abuse, severe bullying and witnessing neighbourhood violence. Participants were classified as having experienced non-complex trauma if they were exposed to trauma that involved a single event or non-interpersonal events only (i.e. their experience met criterion (a), but not all other criteria needed to indicate complex trauma exposure in their lifetime). Examples of non-complex traumas included a one-off assault, an accident and learning about the sudden death of a parent. Two mutually exclusive groups of trauma-exposed participants were therefore formed. Further details are provided in Supplementary Table 1 and Supplementary Fig. 1. Trauma dossiers for each trauma-exposed participant were coded to indicate complex or non-complex trauma exposure by two psychiatrists (interrater reliability of coding for trauma-exposed participants: $\kappa=0.86$ ). Of all participants with available data, 9.1\% (188/2064) reported complex trauma, $21.7 \%(448 / 2064)$ reported non-complex trauma and $0.3 \%(6 / 2064)$ reported trauma but declined to provide a description (excluded from these analyses). 


\section{Psychopathology}

We focused on a measure of general psychopathology, 'p', which was derived by fitting a bi-factor model to 11 symptom scales (PTSD, major depressive disorder, generalised anxiety disorder, disordered eating, attention-deficit hyperactivity disorder (ADHD), conduct disorder, alcohol dependence, cannabis dependence, nicotine dependence, psychotic symptoms and prodromal symptoms) obtained from data collected during interviews conducted at age 18 years, about psychopathology in the previous year. ${ }^{14}$ Scores were scaled to a mean of 100 and s.d. of 15 . To investigate more specific and clinically relevant measures of psychopathology, we also considered the count and occurrence of nine psychiatric disorders (PTSD, major depressive disorder, generalised anxiety disorder, ADHD, conduct disorder, alcohol dependence, cannabis dependence, nicotine dependence and psychotic symptoms) in the previous year, assessed during interviews conducted at age 18 years. Details are provided in Supplementary Table 1.

\section{Cognitive function}

We focused on a measure of general intelligence, IQ, which was tested at age 18 years, using a short version of the Wechsler Adult Intelligence Scale, Fourth Edition. ${ }^{24}$ To investigate more specific aspects of cognitive function, we also considered executive function and processing speed, assessed with subtests of the Cambridge Neuropsychological Test Automated Battery ${ }^{25}$ (including rapid visual information processing (RVP), spatial working memory (SWM) and spatial span subtests) at age 18 years. Scores were scaled to a mean of 100 and s.d. of 15 , and reverse-coded for tests where lower scores indicate better cognitive functioning, so that for all measures of cognitive function lower scores indicate poorer functioning. Details are provided in Supplementary Table 1.

\section{Early childhood vulnerabilities}

Participants were prospectively assessed for early childhood characteristics: internalising and externalising symptoms at age 5 years, IQ score at age 5 years, proportion of family members with a history of mental illness, family socioeconomic status and sex (measures described in Supplementary Table 1). These characteristics were selected for this analysis a priori, to comprehensively capture vulnerabilities known to be associated with trauma exposure, psychopathology and cognitive deficits, ${ }^{26-28}$ which might therefore confound observed associations. Because most trauma-exposed participants reported that their most upsetting trauma occurred during adolescence (age $\geq 12$ years: 529/635 total, 141/187 complex, 388/ 448 non-complex trauma-exposed), and because very-early-life events are unlikely to have been recalled during the trauma interview, these characteristics likely capture pre-trauma vulnerabilities.

\section{Statistical analysis}

To investigate psychopathology and cognitive deficits linked with complex and non-complex trauma in young people, we compared participants exposed to complex trauma or non-complex trauma with trauma-unexposed participants. Additionally, to investigate psychopathology and cognitive deficits uniquely linked with complex trauma within trauma-exposed individuals, we compared participants exposed to complex trauma with those exposed to non-complex trauma.

First, we tested group differences in psychopathology and cognitive function. Next, to map possible confounding factors for the observed associations, we tested whether participants with greater early childhood vulnerability were at higher risk of complex or non-complex trauma exposure. Then, to examine possible confounding effects in the associations of traumas with psychopathology and cognitive deficits, we tested group differences in psychopathology and cognitive function and covaried early childhood vulnerabilities. All of these analyses used generalised estimating equation regression models, accounting for clustering within families. Additionally, we tested whether unobserved genetic or family-wide environmental factors accounted for psychopathology or cognitive deficits linked to complex or non-complex traumas, by examining whether differences in trauma exposure were

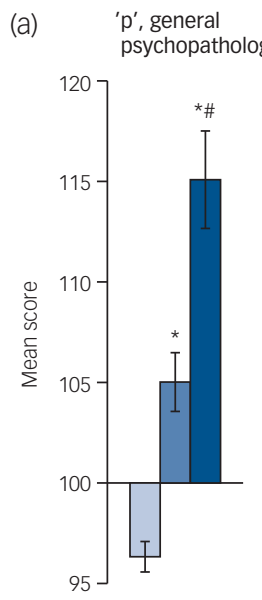

(b) Psychiatric

(c) Psychiatric disorders
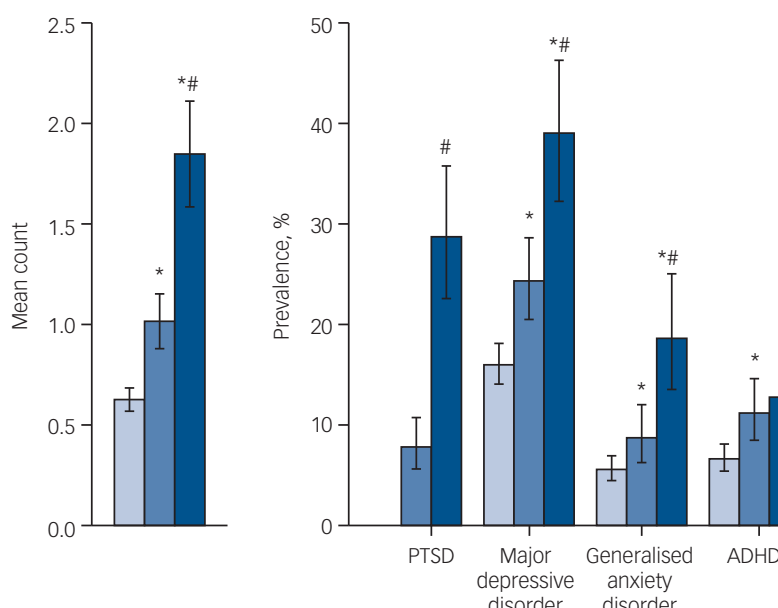

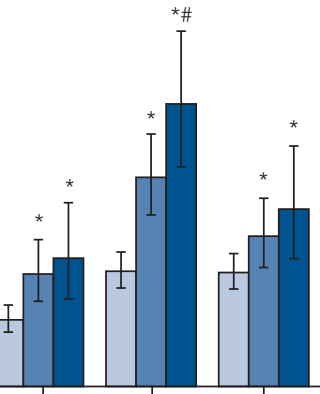

Conduct Alcoho disorder dependence dependence Nicotine Psychotic 
* $P<0.05$ : Complex trauma or non-complex trauma versus no trauma in all participants $\quad \# P<0.05$ : Complex trauma versus non-complex trauma in trauma-exposed participants

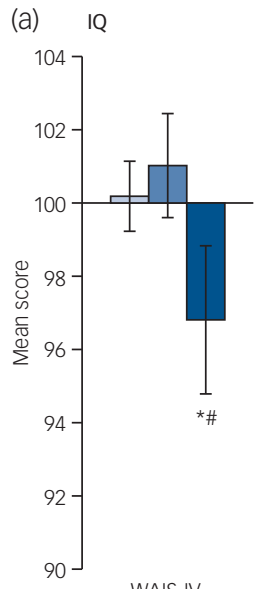

(b) Executive function

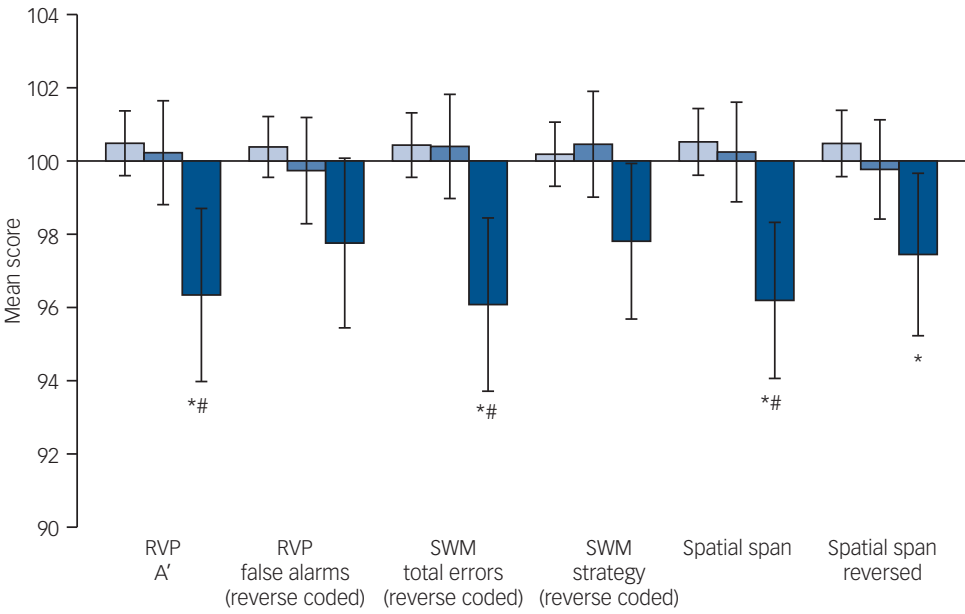

(C) Processing speed

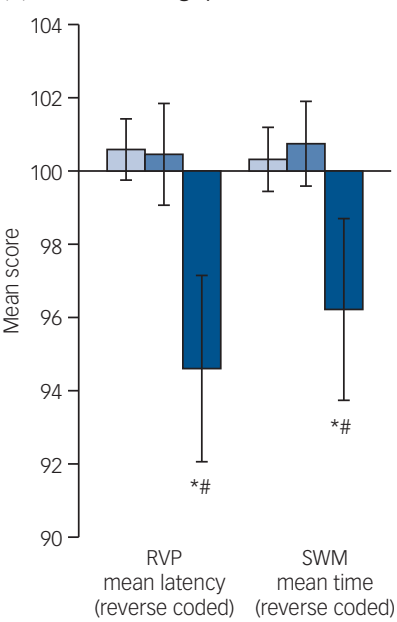

Fig. 2 Trauma exposure and cognitive function. Cognitive function is described in terms of the mean scores of (a) IQ, (b) executive function and (c) processing speed measures in participants exposed to no trauma $(n=1407-1414)$, non-complex trauma $(n=444-447)$ and complex trauma ( $n=185-188)$. Error bars indicate $95 \%$ confidence intervals, with robust s.e. accounting for clustering within families. RVP, rapid visual information processing; SWM, spatial working memory; WAIS-IV, Wechsler Adult Intelligence Scale, Fourth Edition.

correlated with differences in 'p' or IQ score within twin pairs. Further details are provided in the Supplementary Methods.

\section{Results}

\section{Is complex trauma exposure associated with more severe psychopathology?}

We first examined group differences in general psychopathology, 'p', at age 18 years (Fig. 1(a)). Compared with trauma-unexposed participants, those exposed to complex trauma had higher scores of 'p' (regression coefficient $\beta=16.49,95 \%$ CI 13.97-18.96), and so did those exposed to non-complex trauma $(\beta=8.16,95 \% \mathrm{CI}$ 6.72-9.60). Within trauma-exposed participants, those exposed to complex trauma had higher scores of ' $\mathrm{p}$ ' than participants exposed to non-complex trauma $(\beta=9.30,95 \%$ CI 6.47-12.12).

We then considered specific psychiatric disorders (Fig. 1(b) and 1(c)). Compared with trauma-unexposed participants, those exposed to complex trauma had more psychiatric disorders and were more likely to experience each psychiatric disorder assessed. In addition, compared with trauma-unexposed participants, those exposed to non-complex trauma had more psychiatric disorders and were more likely to experience major depressive disorder, generalised anxiety disorder, ADHD, conduct disorder and alcohol dependence. Within trauma-exposed participants, those exposed to complex trauma had more psychiatric disorders and were more likely to experience PTSD, major depressive disorder, generalised anxiety disorder, conduct disorder, cannabis dependence, nicotine dependence and psychotic symptoms than participants exposed to non-complex trauma.

\section{Is complex trauma exposure associated with poorer cognitive function?}

We first examined group differences in general intelligence, determined by IQ score, at age 18 years (Fig. 2(a)). Compared with trauma-unexposed participants, those exposed to complex trauma had lower IQ scores $(\beta=-2.46,95 \%$ CI -4.48 to -0.44$)$. However, participants exposed to non-complex trauma did not have significantly lower IQ scores than those not exposed to trauma $(\beta=0.94$, $95 \%$ CI -0.39 to 2.27 ). Within trauma-exposed participants, those

Table 1 Associations between early childhood vulnerabilities and trauma exposure

$\operatorname{RRR}(95 \% \mathrm{Cl})$

No trauma as base of dependent variable

complex trauma

Internalising symptoms at age 5 years

Externalising symptoms at age 5 years

$1.31(1.13-1.51)$

IQ at age 5 years

$1.28(1.12-1.48)$

Proportion of family members with a history of

$0.95(0.82-1.10)$

mental illness

Lower family socioeconomic status at age 5 years

Female sex

$1.42(1.19-1.68)$

$1.75(1.42-2.15)$

$1.75(1.25-2.45)$

Non-complex trauma

Internalising symptoms at age 5 years

Externalising symptoms at age 5 years

IQ at age 5 years

Proportion of family members with a history of mental illness

Lower family socioeconomic status at age 5 years Female sex

Non-complex trauma as base of dependent variable

complex trauma

Internalising symptoms at age 5 years

Externalising symptoms at age 5 years

IQ at age 5 years

Proportion of family members with a history of mental illness

Lower family socioeconomic status at age 5 years Female sex

$0.96(0.85-1.08)$

$1.05(0.94-1.18)$

$0.99(0.89-1.11)$

1.20 (1.07-1.35)

$1.01(0.87-1.16)$

$0.98(0.78-1.23)$

$1.37(1.16-1.62)$

$1.22(1.04-1.42)$

$0.95(0.81-1.13)$

$1.18(0.97-1.42)$

$1.74(1.38-2.19)$

$1.79(1.23-2.59)$

Unadjusted relative risk ratios (RRR) with $95 \%$ confidence intervals are presented for associations between early childhood vulnerabilities and trauma exposure in all participants $(n=2004-2058)$. We provide results of multinomial models with no trauma as the cipants $(n=2004-2058)$. We provide results of multinomial models with no trauma as the base of the dependent variable, and with non-complex trauma as the base of the dependent variable (results for no trauma over non-complex trauma have been omitted, as the more meaningful results for non-complex trauma over no trauma have been provided). Early childhood vulnerabilities measured on a continuous scale had been standardised to a mean of 0 and s.d. of 1 (internalising and externalising symptoms at age 5 years, IQ at age 5 years and proportion of family members with a history of mental illness). Bold text signifies $P<0.05$ 


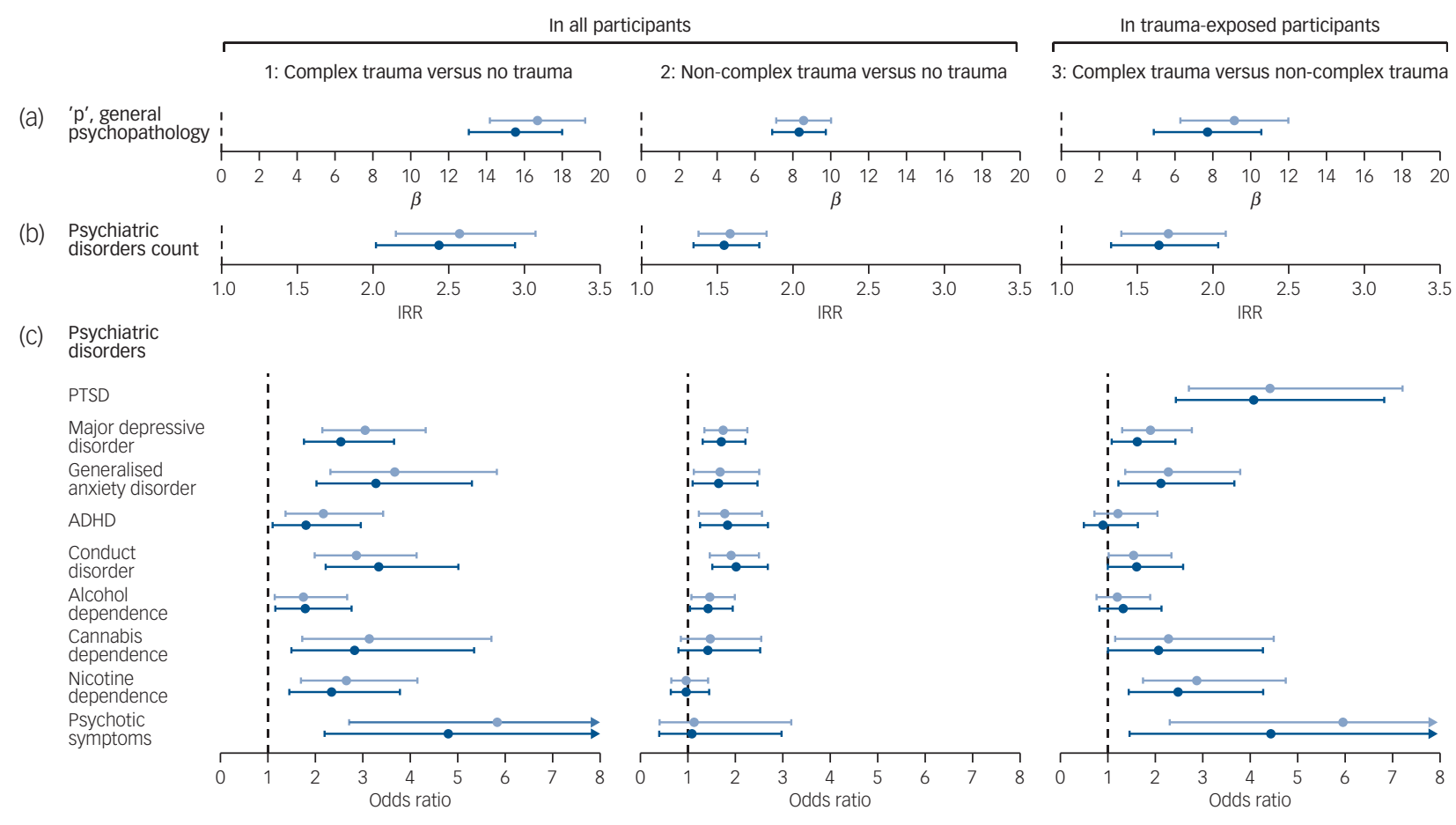

Fig. 3 Associations between trauma exposure and psychopathology, including controlling for early childhood vulnerabilities. (a) Linear regression coefficients ( $\beta$ ), (b) incidence rate ratios and (c) odds ratios with $95 \%$ confidence intervals (bars) are presented for associations between trauma exposure and psychopathology in all participants $(n=1965-1990)$, i.e. those exposed to complex trauma versus no trauma (column 1) and non-complex trauma versus no trauma (column 2); and in trauma-exposed participants $(n=613-620)$, i.e. those exposed to complex trauma versus non-complex trauma (column 3). We present results from univariable (unadjusted) models (lighter colour), and multivariable models adjusted for the effects of early childhood vulnerabilities (darker colour). Dashed lines are lines of no difference. ADHD, attention-deficit hyperactivity disorder; IRR, incidence rate ratio; PTSD, post-traumatic stress disorder

exposed to complex trauma had lower IQ scores than participants exposed to non-complex trauma $(\beta=-4.55,95 \% \mathrm{CI}-6.89$ to -2.20$)$.

We then considered executive function and processing speed (Fig. 2(b) and 2(c)). Compared with trauma-unexposed participants, those exposed to complex trauma had poorer functioning on several measures of executive function (RVP A', SWM errors, spatial span and spatial span reversed) and on both measures of processing speed. However, participants exposed to non-complex trauma did not have significantly different scores on measures of these cognitive functions compared with participants not exposed to trauma. Within trauma-exposed participants, those exposed to complex trauma had poorer functioning on several measures of executive function (RVP A', SWM errors and spatial span) and on both measures of processing speed than participants exposed to non-complex trauma.

\section{Is greater early childhood vulnerability associated with higher risk of complex trauma exposure?}

Most of the a priori selected early childhood vulnerabilities were associated with complex trauma exposure over no trauma exposure. In contrast, the only early vulnerability significantly associated with non-complex trauma exposure over no trauma exposure was family history of mental illness. Likewise, most early vulnerabilities were associated with complex trauma exposure over non-complex trauma exposure (Table 1).

\section{Do early childhood vulnerabilities account for psychopathology linked to complex trauma?}

We found that early childhood vulnerabilities did not explain the associations of complex or non-complex trauma exposure with psychopathology. The association between complex trauma (versus no trauma) and 'p' did not substantially change after accounting for all early childhood vulnerabilities ( $\beta$ reduced in magnitude by $7.0 \%$ and remained statistically significant) (Fig. 3(a) column 1 , and Supplementary Table 2(a)). Similarly, the association between non-complex trauma (versus no trauma) and 'p' did not substantially change after early childhood vulnerabilities were taken into account ( $\beta$ reduced in magnitude by $2.9 \%$ and remained statistically significant) (Fig. 3(a) column 2, and Supplementary Table 2(a)). Within trauma-exposed participants, the association between complex trauma (versus non-complex trauma) and 'p' did not substantially change after accounting for early childhood vulnerabilities ( $\beta$ reduced in magnitude by $15.5 \%$ and remained statistically significant) (Fig. 3(a) column 3, and Supplementary Table 2(a)). Twin analyses found consistent results. Within pairs of twins who grew up in the same family environment and share some (DZ twins) or all (MZ twins) of their genetic material, participants exposed to complex trauma had higher scores of ' $\mathrm{p}$ ' than their co-twin not exposed to complex trauma. This indicates that complex trauma is associated with 'p' independent of family environment and genetic risk (Supplementary Table 3). When considering the count and occurrence of psychiatric disorders, similar results were also found (Fig. 3(b) and 3(c) columns 1-3, and Supplementary Table 2(b) and 2(c)).

\section{Do early childhood vulnerabilities account for cognitive deficits linked to complex trauma?}

We found that early childhood vulnerabilities largely accounted for the associations of complex trauma with lower IQ score and poorer executive function, but not the associations with slower processing 
In all participants

(a) IQ

WAIS-IV

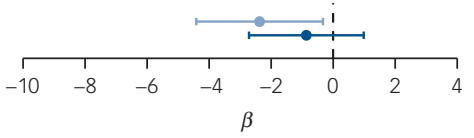

(b) Executive function

RVP A'

RVP false alarms

(reverse coded)

SWM total errors

(reverse coded)

SWM strategy

(reverse coded)

Spatial span

Spatial span

reversed

(C) Processing speed

RVP mean latency (reverse coded)

sWM mean time (reverse coded)
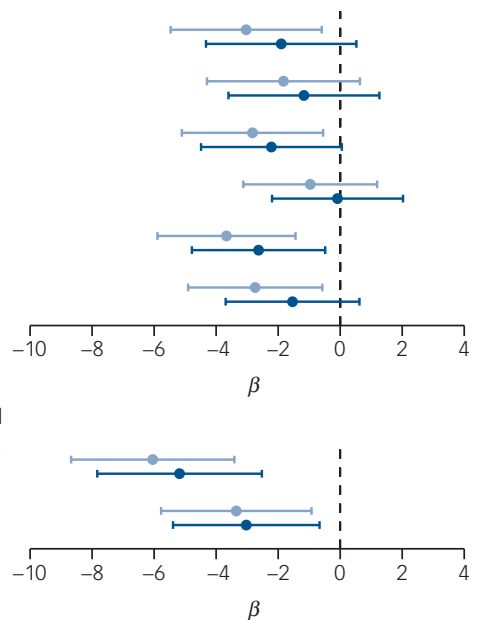

2: Non-complex trauma versus no trauma
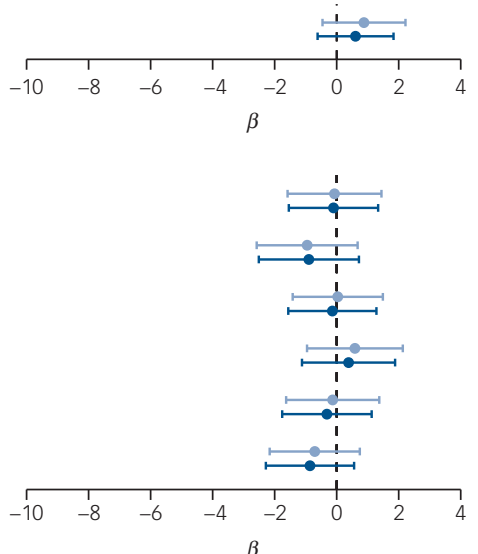

$\beta$

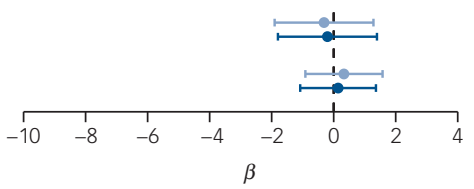

In trauma-exposed participants

3: Complex trauma versus non-complex trauma
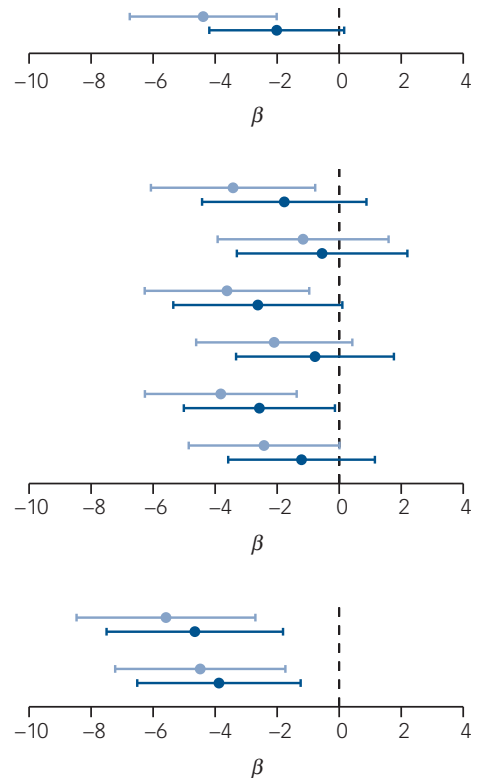

Fig. 4 Associations between trauma exposure and cognitive function, including controlling for early childhood vulnerabilities. Linear regression coefficients $(\beta)$ with $95 \%$ confidence intervals (bars) are presented for associations between trauma exposure and cognitive function in all participants ( $n=1973-1983)$, i.e. those exposed to complex trauma versus no trauma (column 1 ) and non-complex trauma versus no trauma (column 2); and in trauma-exposed participants $(n=614-618)$, i.e. those exposed to complex trauma versus non-complex trauma (column 3). We present results from univariable (unadjusted) models (lighter colours), and multivariable models adjusted for the effects of early childhood vulnerabilities (darker colours). Dashed lines are lines of no difference. RVP, rapid visual information processing; SWM, spatial working memory; WAIS-IV, Wechsler Adult Intelligence Scale, Fourth Edition.

speed. The association between complex trauma (versus no trauma) and IQ score was substantially attenuated after early childhood vulnerabilities were taken into account ( $\beta$ reduced in magnitude by $63.6 \%$ and became statistically non-significant) (Fig. 4(a) column 1, and Supplementary Table 4(a)). As in unadjusted analyses, non-complex trauma (versus no trauma) was not associated with IQ score after accounting for early childhood vulnerabilities (Fig. 4(a) column 2, and Supplementary Table 4(a)). Within trauma-exposed participants, the association between complex trauma (versus non-complex trauma) and IQ score was substantially attenuated after accounting for early childhood vulnerabilities ( $\beta$ reduced in magnitude by $54.1 \%$ and became statistically non-significant) (Fig. 4(a) column 3, and Supplementary Table 4(a)). Twin analyses found consistent results. Within twin pairs who grew up in the same family environment and share genetic material, complex trauma was not correlated with IQ score. This suggests that family environment and genetic factors likely explain the associations observed at the individual level (Supplementary Table 5). When considering executive function and processing speed, early childhood vulnerabilities accounted for the associations of complex trauma (versus no trauma) with some measures of executive function (RVP A', SWM errors and spatial span reversed), but not processing speed (Fig. 4(b) and 4(c) column 1, and Supplementary Table 4(b) and 4(c)). Finally, within traumaexposed participants, early childhood vulnerabilities accounted for the associations of complex trauma (versus non-complex trauma) with some measures of executive function (RVP A' and SWM errors), but not processing speed (Fig. 4(b) and 4(c) column 3, and Supplementary Table 4(b) and 4(c)).

\section{Discussion}

We found that young people exposed to complex traumas had more severe psychopathology and poorer cognitive function compared with trauma-unexposed peers and those exposed to non-complex traumas. Although their psychopathology was considerably more severe, their cognitive deficits were relatively modest on average. Early childhood vulnerabilities had an important role in these presentations, as they predicted later exposure to complex trauma and largely explained the associations between complex trauma and cognitive deficits. Nevertheless, psychopathology remained associated with complex trauma even after accounting for early childhood vulnerabilities. These findings from a large, population-based cohort strengthen and extend the limited empirical evidence in this area, ${ }^{10-13}$ and caution that conflating complex and non-complex traumas in research and clinical practice has likely led to an underestimation of psychopathology, cognitive deficits and pre-existing vulnerabilities linked with complex trauma.

\section{Limitations}

Our findings should be interpreted in light of some limitations. First, lifetime trauma exposure was measured by retrospective self-report at age 18 years, when psychopathology and cognitive function were also assessed. As such, it is possible that our results partly reflect common method bias. However, these findings based on self-reported complex trauma are consistent with those from studies of victimisation measured prospectively or through informants, ${ }^{14,15}$ suggesting that common method bias is unlikely 
to fully explain our results. Regardless of the underlying mechanisms, our findings suggest that self-reports of complex trauma can pragmatically be used to identify a group of individuals with greater mental health needs than peers reporting non-complex trauma or no trauma. Second, our findings may only be valid within the boundaries of our measures. For example, complex traumas in pre-school years (unlikely to be retrospectively recalled and possibly masked by our adjustment for vulnerabilities at age 5 years) or complex trauma types not observed in our study (e.g. living in conflict areas) may have different sequelae. Furthermore, it is unclear if patterns similar to the ones described here would apply to other aspects of psychopathology (e.g. complex PTSD, personality disorders or autism spectrum disorders) or cognitive functions (e.g. reward or threat processing) not included in our study. Third, findings from our twin sample might not generalise to singletons. However, the prevalence of trauma and psychiatric disorders and the strength of the associations observed in our sample are similar to those found in samples of singletons, ${ }^{29}$ supporting the generalisability of our results. Despite these limitations, our findings have implications for research and clinical practice.

\section{Implications}

Our findings demonstrate that individuals exposed to complex trauma have greater mental health needs than those exposed to non-complex trauma, since they experience more severe psychopathology and poorer cognitive function. These findings may reflect qualitative differences between complex and non-complex trauma as originally proposed, ${ }^{4-7}$ or may reflect quantitative differences where complex traumas are at the upper end of a trauma continuum, for instance because of high severity or frequency. Nevertheless, underappreciation of these differences in trauma research and clinical practice has likely led to underrecognition of the greater mental health needs of people exposed to complex traumas. Research that further explores these differences will therefore be valuable to better understand and address trauma-related mental health needs.

Individuals exposed to non-complex trauma have less severe psychopathology than those exposed to complex trauma, but they nevertheless have more severe psychopathology than trauma-unexposed peers. The higher risk of psychopathology in people exposed to non-complex trauma is not limited to PTSD, as originally implied, ${ }^{4-7}$ but extends across several internalising and externalising disorders. Furthermore, because non-complex trauma has higher prevalence than complex trauma, non-complex trauma contributes to a large number of cases of trauma-related psychopathology in the population.

To map the broad mental health needs of people exposed to trauma, and particularly complex trauma, it is therefore necessary to undertake comprehensive assessments that evaluate wideranging psychopathology and cognitive deficits, both in research and clinical practice. Furthermore, research is needed to understand how to address these needs most effectively, including investigating whether broader treatment approaches might be more helpful than standard treatments, and whether these might be more useful for individuals exposed to complex trauma compared with those exposed to non-complex trauma. Such treatments might involve phase-based approaches that target symptoms sequentially, ${ }^{30,31}$ or trans-diagnostic approaches that target common mechanisms underlying the cooccurrence of disorders. ${ }^{32}$

Our findings also indicate that pre-existing vulnerabilities predict complex trauma, but not non-complex trauma exposure, and largely explain associations between complex trauma and cognitive deficits. These findings challenge the hypothesis that complex trauma leads to effects on the brain detectable through cognitive deficits, ${ }^{16}$ and therefore warn against simplistic causal interpretations of associations in research and clinical case formulation. ${ }^{17}$ Additionally, our findings indicate that pre-existing vulnerabilities do not explain psychopathology linked with complex trauma. These findings suggest that features of the trauma (e.g. nature, severity, recurrence or timing) or responses to the trauma (e.g. maladaptive cognitions, behavioural coping strategies or emotion processing) could lead to psychopathology. Further research that provides a better understanding of these underlying mechanisms could inform the development of new, more effective interventions for mental illness in people exposed to complex trauma. ${ }^{17}$

Finally, our findings are consistent with our team's research on victimisation, ${ }^{14,15}$ suggesting that despite differences in these constructs (Supplementary Fig. 1), both capture exposures related to psychopathology and cognitive deficits, which have similar origins. These findings highlight the importance of reconciling nomenclature and measurement in these related fields to most effectively inform research and clinical progress.

Stephanie J. Lewis $\mathbb{D}, \mathrm{MBBS}$, MRCPsych, Department of Child and Adolescent

Psychiatry, Institute of Psychiatry, Psychology and Neuroscience, King's College London,

UK; Karestan C. Koenen, PhD, Department of Epidemiology, Harvard T.H. Chan School

of Public Health, USA; Antony Ambler, MSc, Social, Genetic and Developmental

Psychiatry Centre, Institute of Psychiatry, Psychology and Neuroscience, King's College

London, UK; Louise Arseneault, PhD, Social, Genetic and Developmental Psychiatry

Centre, Institute of Psychiatry, Psychology and Neuroscience, King's College London, UK;

Avshalom Caspi, PhD, Social, Genetic and Developmental Psychiatry Centre, Institute

of Psychiatry, Psychology and Neuroscience, King's College London, UK; Department of Psychology and Neuroscience, Duke University, USA; Department of Psychiatry and Behavioral Sciences, Duke University, USA; and Center for Genomic and Computational Biology, Duke University, USA; Helen L. Fisher (D), PhD, Social, Genetic and

Developmental Psychiatry Centre, Institute of Psychiatry, Psychology and Neuroscience, King's College London, UK; and ESRC Centre for Society and Mental Health, King's college London, UK; Terrie E. Moffitt, PhD, Social, Genetic and Developmental Psychiatry Centre, Institute of Psychiatry, Psychology and Neuroscience, King's College London, UK; Department of Psychology and Neuroscience, Duke University, USA; Department of Psychiatry and Behavioral Sciences, Duke University, USA; and Center for Genomic and Computational Biology, Duke University, USA; Andrea Danese (D), MD, PhD,

Department of Child and Adolescent Psychiatry, Institute of Psychiatry, Psychology and Neuroscience, King's College London, UK; Social, Genetic and Developmental Psychiatry Centre, Institute of Psychiatry, Psychology and Neuroscience, King's College London, UK; and National and Specialist CAMHS Clinic for Trauma, Anxiety, and Depression, South London and Maudsley NHS Foundation Trust, UK

Correspondence: Andrea Danese. Email: andrea.danese@kcl.ac.uk

First received 8 Dec 2020, final revision 09 Apr 2021, accepted 13 Apr 2021

\section{Supplementary material}

To view supplementary material for this article, please visit https://doi.org/10.1192/bjp.2021.57.

\section{Data availability}

E-Risk study data is available to researchers upon successful application for access, according to the E-Risk study governance processes (eriskstudy.com).

\section{Acknowledgements}

We are grateful to the study mothers and fathers, the twins and their teachers for their participation, and to members of the E-Risk team for their dedication, hard work and insights. We thank the Young Person's Mental Health Advisory Group from the National Institute for Health Research Maudsley Biomedical Research Centre at South London and Maudsley NHS Foundation Trust and King's College London for their feedback, which helped shape these research plans. We are also grateful to Dr Jia Ying Teng, who assisted with coding trauma variables.

\section{Author contributions}

S.J.L. and A.D. formulated the research questions and designed the analysis plan. K.C.K., L.A., A.C., H.L.F. and T.E.M. contributed to the analysis plan. S.J.L. coded the trauma variables and analysed the data. A.A. checked the analysis for reproducibility. S.J.L. and A.D. drafted the manuscript. All authors assisted with interpretation, commented on drafts of the manuscript and approved the final version. 


\section{Funding}

The E-Risk study is funded by the UK Medical Research Council (MRC; grant G1002190). Additional support was provided by the US National Institute of Child Health and Development (grant HD077482), the Jacobs Foundation, the UK National Society for Prevention of Cruelty to Children and the UK Economic and Social Research Council (ESRC) S.J.L. is supported by an MRC Clinical Research Training Fellowship and received a UK Roya College of Psychiatrists Margaret Slack Fellowship. L.A. is the Mental Health Leadership Fellow for the ESRC. H.L.F. receives salary support from the ESRC Centre for Society and National Institute for Health Research (NIHR) Biomedical Research Centre at South London and Maudsley NHS Foundation Trust and King's College London. The views expressed are those of the authors and not necessarily those of the NHS, the NIHR, the Department of Health and Social Care or the ESRC.

\section{Declaration of interest}

\section{References}

1 American Psychiatric Association. Diagnostic and Statistical Manual of Mental Disorders (5th edn). American Psychiatric Association, 2013.

2 Andreasen NC. Acute and delayed posttraumatic stress disorders: a history and some issues. Am J Psychiatry 2004; 161: 1321-3.

3 Ehlers A, Clark DM. A cognitive model of posttraumatic stress disorder. Behav Res Ther 2000; 38: 319-45

4 Terr LC. Childhood traumas: an outline and overview. Am J Psychiatry 1991; 148: $10-20$.

5 Herman JL. Complex PTSD: a syndrome in survivors of prolonged and repeated trauma. J Trauma Stress 1992; 5: 377-91.

6 Pelcovitz D, van der Kolk B, Roth S, Mandel F, Kaplan S, Resick P, et al. Development of a criteria set and a structured interview for disorders of extreme stress (SIDES). J Trauma Stress 1997; 10: 3-16.

7 Cook A, Blaustein M, Spinazzola J, van der Kolk B, editors. Complex Trauma in Children and Adolescents. National Child Traumatic Stress Network Complex Trauma Task Force, 2003 (https://www.nctsn.org/resources/complex-traumachildren-and-adolescents).

8. Maercker A, Brewin CR, Bryant RA, Cloitre M, van Ommeren M, Jones LM, et al Diagnosis and classification of disorders specifically associated with stress: proposals for ICD-11. World Psychiatry 2013; 12: 198-206.

9 van der Kolk BA. Developmental trauma disorder: toward a rational diagnosis for children with complex trauma histories. Psychiatr Ann 2005; 35: 401-8.

10 van der Kolk BA, Roth S, Pelcovitz D, Sunday S, Spinazzola J. Disorders of extreme stress: the empirical foundation of a complex adaptation to trauma. J Trauma Stress 2005; 18: 389-99.

11 Cloitre M, Garvert DW, Brewin CR, Bryant RA, Maercker A. Evidence for proposed ICD-11 PTSD and complex PTSD: a latent profile analysis. Eur J Psychotraumatology 2013; 4: 20706.

12 Wamser-Nanney R, Vandenberg BR. Empirical support for the definition of a complex trauma event in children and adolescents. J Trauma Stress 2013; 26 : 671-8.

13 op den Kelder R, Ensink JBM, Overbeek G, Maric M, Lindauer RJL. Executive function as a mediator in the link between single or complex trauma and posttraumatic stress in children and adolescents. Qual Life Res 2017; 26: 1687-96.
14 Schaefer JD, Moffitt TE, Arseneault L, Danese A, Fisher HL, Houts R, et al Adolescent victimization and early-adult psychopathology: approaching causal inference using a longitudinal twin study to rule out noncausal explanations. Clin Psychol Sci 2018; 6: 352-71.

15 Danese A, Moffitt TE, Arseneault L, Bleiberg BA, Dinardo PB, Gandelman SB et al. The origins of cognitive deficits in victimized children: implications for neuroscientists and clinicians. Am J Psychiatry 2017; 174: 349-61.

16 Danese A, Lewis SJ. Psychoneuroimmunology of early-life stress: the hidden wounds of childhood trauma? Neuropsychopharmacology 2017; 42: 99-114.

17 Danese A. Annual research review: rethinking childhood trauma-new research directions for measurement, study design and analytical strategies. J Child Psychol Psychiatry 2020; 61: 236-50.

18 Silberg JL, Copeland W, Linker J, Moore AA, Roberson-Nay R, York TP, et al. Psychiatric outcomes of bullying victimization: a study of discordant monozygotic twins. Psychol Med 2016; 46: 1875-83.

19 Dinkler L, Lundström S, Gajwani R, Lichtenstein P, Gillberg C, Minnis H, et al. Maltreatment-associated neurodevelopmental disorders: a co-twin control analysis. J Child Psychol Psychiatry 2017; 58: 691-701.

20 Trouton A, Spinath FM, Plomin R. Twins Early Development Study (TEDS): a multivariate, longitudinal genetic investigation of language, cognition and behavior problems in childhood. Twin Res 2002; 5: 444-8.

21 Moffitt TE, the E-Risk Study Team. Teen-aged mothers in contemporary Britain. J Child Psychol Psychiatry 2002; 43: 727-42.

22 Odgers CL, Caspi A, Russell MA, Sampson RJ, Arseneault L, Moffitt TE, et al. Supportive parenting mediates neighborhood socioeconomic disparities in children's antisocial behavior from ages 5 to 12. Dev Psychopathol 2012; 24: 705-21.

23 Fisher HL, Caspi A, Moffitt TE, Wertz J, Gray R, Newbury J, et al. Measuring adolescents' exposure to victimization: the Environmental Risk (E-Risk) Longitudinal Twin Study. Dev Psychopathol 2015; 27: 1399-416.

24 Wechsler D. Wechsler Adult Intelligence Scale (4th edn). Pearson, 2008.

25 Cambridge Cognition. CANTAB Eclipse Test Administration Guide. Cambridge Cognition, 2006.

26 Koenen KC, Moffitt TE, Poulton R, Martin J, Caspi A. Early childhood factors associated with the development of post-traumatic stress disorder: results from a longitudinal birth cohort. Psychol Med 2007; 37: 181-92.

27 Caspi A, Houts RM, Belsky DW, Goldman-Mellor SJ, Harrington H, Israel S, et al. The $\mathrm{p}$ factor: one general psychopathology factor in the structure of psychiatric disorders? Clin Psychol Sci 2014; 2: 119-37.

28 Deary IJ, Pattie A, Starr JM. The stability of intelligence from age 11 to age 90 years: the Lothian birth cohort of 1921. Psychol Sci 2013; 24: 2361-8.

29 Lewis SJ, Arseneault L, Caspi A, Fisher HL, Matthews T, Moffitt TE, et al. The epidemiology of trauma and post-traumatic stress disorder in a representative cohort of young people in England and Wales. Lancet Psychiatry 2019; 6: 247-56.

30 Cloitre M, Koenen KC, Cohen LR, Han H. Skills training in affective and interpersonal regulation followed by exposure: a phase-based treatment for PTSD related to childhood abuse. J Consult Clin Psychol 2002; 70: 1067-74.

31 Cohen JA, Mannarino AP, Kliethermes M, Murray LA. Trauma-focused CBT for youth with complex trauma. Child Abuse Negl 2012; 36: 528-41.

32 Dalgleish T, Black M, Johnston D, Bevan A. Transdiagnostic approaches to mental health problems: current status and future directions. J Consult Clin Psychol 2020; 88: 179-95. 